\title{
Gesundheitsversorgung im Jahr 2030 - Spitäler und ärztliche Berufsbildung sind gefordert
}

In allen europäischen Gesundheitssystemen müssen die Personalressourcen heute und vermehrt in den kommenden Jahren effizienter eingesetzt werden. Die Europäische Kommission hat deshalb eine Arbeitsgruppe beauftragt, bis Ende 2008 ein «Greenpaper» zum Thema « Workforce in Healthcare» zu erarbeiten. Darin soll auch die Migration der Ärztinnen und Ärzte thematisiert werden.

Das Schweizer Gesundheitssystem ist diesbezüglich seit längerem gefordert. So sollten die Spitäler die Bewirtschaftung der ärztlichen Ressourcen, mit denen in den letzten Jahren (begründet mit der Einführung der 50-Stunden-Woche) grosszügig umgegangen wurde, effizienter gestalten. Eine blosse Steigerung der Anzahl Stellen für Assistenzärzte in Weiterbildung kann nicht die Lösung sein. Diese Stellen wurden zwischen 2004 und 2008 von 8229 auf 9009 erhöht. Schaut man genauer hin, zeigt sich, dass in diesem Zeitraum die Zahl der durch Ärztinnen und Ärzte mit Schweizer Diplom besetzten Stellen um 280 abnahm, während bei den mit ausländischen Diplomträgern besetzten Stellen eine Zunahme um 1060 zu verzeichnen war.

So lange die Schweizer Spitäler ihre Attraktivität im europäischen Markt, namentlich in der ärztlichen Weiterbildung, behalten, kann im Prinzip so weiter gewirtschaftet werden. Die von der Kommission für Weiter- und Fortbildung eingeleiteten Massnahmen zur Optimierung der Weiterbildung (Assistentenumfrage, arbeitsplatzbasiertes Assessment, Visitationen, Logbücher) tragen zum Erhalt dieser Attraktivität bei. Die aktuellen Strukturprobleme innerhalb der Spitallandschaft müssen von Ärzteschaft und Spitalträgern gemeinsam gelöst werden. Es wäre falsch, darauf zu setzen, dass sich diese Probleme mit der Einführung der DRG «von selbst» lösen werden.

Daneben sollen die demographischen und epidemiologischen Probleme der Gesundheitsversorgung angegangen werden. Auch hier geht es um die Bewirtschaftung der Humanressourcen - und zwar aller Gesundheitsberufe, im Spital wie in der Ambulanz. Die Verteilung der Ärzte in den Institutionen und Regionen muss entsprechend den Bedürfnissen der Bevölkerung erfolgen. Nur so kann die Versorgungssicherheit in allen Regionen in einer Zeit erhalten bleiben, die durch «zentripetale Tendenzen» in Bezug auf die Lebensgestaltung gekennzeichnet ist.

In dieser Ausgabe der Schweizerischen Ärztezeitung wird in einem Artikel auf die Notwendigkeit neuer Ver- sorgungsmodelle und Kompetenzen hingewiesen [1]. Die bisherigen Diskussionen um das Arztbild der Zukunft sind zu vertiefen, sowohl in den Institutionen als auch in den Fachgesellschaften. Damit die Patientenversorgung optimal gestaltet wird, sind alle daran beteiligten Gesundheitsberufe, namentlich die Pflegeberufe und klinischen Psychologen, in die Diskussion einzubeziehen.

Die Entlastung der Ärzte von administrativen Aufgaben ist eine alte Forderung. Der gezielte Einsatz von Informations- und Kommunikationstechnologie kann helfen, genügt alleine aber nicht. Das Delegieren bisher von Ärzten durchgeführter, aber nicht zwingend ärztlicher Tätigkeiten an andere Gesundheitsberufe ist ein weiterer sinnvoller, möglicherweise sogar unumgänglicher Lösungsansatz. Die Fachgesellschaften sind hier besonders gefordert: Sie sollten die Weiterbildungsprogramme vermehrt auf die Bedürfnisse der Bevölkerung und die Lernziele auf die ärztlichen Kernkompetenzen ausrichten. Bei allen Lernzielen ist zu beurteilen, ob eine ärztliche Kompetenz zur Erfüllung der angestrebten Aufgabe bzw. Gesundheitsleistung notwendig ist oder nicht. Auch in der Fortbildung sind solche Überlegungen anzustellen. Die FMH und die Fachgesellschaften können mittels eines zielgerichteten Fortbildungsportfolios und spezifischer Assessmentmethoden ihre Mitglieder unterstützen.

Eine sichere und qualitativ hochstehende Versorgung der Patienten bleibt auch im Jahr 2030 gewährleistet. Dafür ist ein gemeinsames und kooperatives Engagement der Ärzteschaft und Vertreter weiterer Gesundheitsberufe notwendig. Mit einer zielgerichteten und effizienten ärztlichen Berufsbildung ist die Attraktivität des Arztberufes zu erhalten bzw. zu erhöhen; ein besonderes Augenmerk gilt der Grundversorgung und der Psychiatrie. Diese Ziele verfolgen wir bereits heute.

Dr. med. Max Giger, Mitglied des Zentralvorstands und Präsident der Kommission für Weiter- und Fortbildung der FMH

1 Giger M, De Geest S. Neue Versorgungsmodelle und Kompetenzen sind gefragt. Schweiz Ärztezeitung. 2008; 89(43):1839-43. 